

\title{
The Survival Assumption in Intertemporal Economies
}

Jean-Marc Bonnisseau, Alexandrine Jamin

\section{To cite this version:}

Jean-Marc Bonnisseau, Alexandrine Jamin. The Survival Assumption in Intertemporal Economies. 2009. halshs-00435256

\section{HAL Id: halshs-00435256 \\ https://shs.hal.science/halshs-00435256}

Submitted on 24 Nov 2009

HAL is a multi-disciplinary open access archive for the deposit and dissemination of scientific research documents, whether they are published or not. The documents may come from teaching and research institutions in France or abroad, or from public or private research centers.
L'archive ouverte pluridisciplinaire HAL, est destinée au dépôt et à la diffusion de documents scientifiques de niveau recherche, publiés ou non, émanant des établissements d'enseignement et de recherche français ou étrangers, des laboratoires publics ou privés. 


\section{Documents de Travail du Centre d'Economie de la Sorbonne}

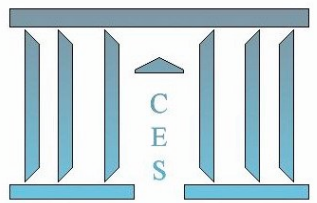

The Survival Assumption in Intertemporal Economies

Jean-Marc BonNISSEAU, Alexandrine JAMIN

2009.76

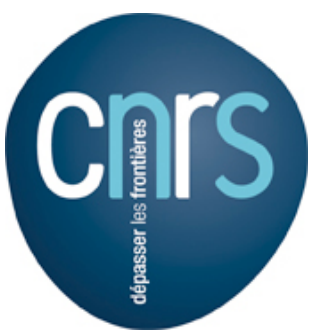

Maison des Sciences Économiques, 106-112 boulevard de L'Hôpital, 75647 Paris Cedex 13 http://ces.univ-paris1.fr/cesdp/CES-docs.htm 


\title{
The Survival Assumption in Intertemporal Economies
}

\author{
J.M. Bonnisseau and A. Jamin ${ }^{1}$
}

November 2009

\begin{abstract}
In an economy with a non-convex production sector, we provide an assumption on each individual producer, which implies that the survival assumption holds true at the aggregate level for general pricing rules. For the marginal pricing rule, we derive this assumption from the bounded marginal productivity of inputs. We apply this approach to intertemporal economies and we show how our assumption fits well with the time structure leading to a tractable existence result of equilibria, which could be apply to discrete dynamical growth models.
\end{abstract}

Keywords General Equilibrium Theory, Increasing Returns, Survival Assumption, Marginal Pricing, General Pricing Rules.

JEL Classification: C62, C67, D21, D51

\section{Introduction}

The presence of increasing returns to scale, of fixed costs, and, more generally the lack of convexity in the production sector, are recognized as a failure of the competitive mechanism. Walras (1874) first proposed that the non convex firms should be set to follow an average cost pricing behaviour. Later, the theory of marginal cost pricing has been developed, with the works of Pigou (1932), Lange (1936, 1937), Lerner (1936), Hotelling (1938), and later of Allais (1953). Since Guesnerie (1975), this theory has been formulated in the general framework of general equilibrium theory, and many results, addressing as well the existence problem of marginal cost pricing equilibria as their optimality, has been published since. In parallel, another approach was developed, with the emergence of existence results for general pricing rules with bounded losses. For a global survey, see the special issue of the

\footnotetext{
${ }^{1}$ Paris School of Economics, Université Paris 1 Panthéon-Sorbonne, CNRSUMR 8174, Centre d'Economie de la Sorbonne 106-112 Boulevard de l'Hôpital 75647 Paris cedex 13, France, Jean-Marc.Bonnisseau@univ-paris1.fr, Alexandrine. Jamin@univ-paris1.fr
} 
Journal of Mathematical Economics on General Equilibrium and Increasing Returns (See, Cornet 1988, Brown's chapter in the Handbook of Mathematical Economics 1992, or Villar 1999).

Beside standard assumptions on the consumers and producers, the existence results require a crucial assumption, namely the survival assumption, which is sufficient for the marginal pricing rule and for the bounded losses general pricing rules. The purpose of this hypothesis is to solve the aggregate survival problem. In the classical Arrow-Debreu framework, it is sufficient to assume that the total initial endowments vector is positive and the inactivity is feasible for each firms. Then, the Survival Assumption is satisfied when the firms are price-taker and profit maximizer. In models incorporating increasing returns, where firms may exhibit losses, this kind of assumption is no longer enough.

Let us present more precisely the Survival Assumption. In an economy with finite numbers, $\ell$ of commodities, and $n$ of firms, we denote by $Y_{j}$ the production set of firm $j$ and by $\varphi_{j}$ its pricing rule, that associates to any production $y_{j} \in Y_{j}$ a set $\varphi_{j}\left(y_{j}\right)$ of price systems compatible with $y_{j}$, according to the pricing behaviour of firm $j$. Firm $j$ is thus said to be at equilibrium for a pair $\left(p, y_{j}\right)$ if the price system $p$ is acceptable for the firm $j$ at $y_{j} \in Y_{j}$, i.e. $p \in \varphi_{j}\left(y_{j}\right)$. A production equilibrium is then a state $\left(p,\left(y_{j}\right)\right)$ of the economy in which the price system $p$ is acceptable for each firm, according to its pricing behaviour, for the given productions $\left(y_{j}\right)$. Bonnisseau and Cornet (1991) propose the following version of the survival assumption: at production equilibrium, if a positive amount of a reference commodity bundle is added to the total initial endowments, so that the production equilibrium becomes attainable, then the total wealth in the economy is above the consumers aggregate subsistence level. This kind of assumption is unsatisfactory in the sense that it states a condition on the set of production equilibria, an endogenous variable of the model. It would be worth preferable to state a condition on the individual primitive data of the economy, notably on the firms characteristics, which does not involve any coordination among the producers.

In the case of marginal pricing, Vohra (1992) proposes a conditions, called bounded marginal return, which generalizes a result in Bonnisseau-Cornet (1990). The intuition comes from the single consumer, single producer, two commodities economy. In that case, the Survival Assumption may not be 
satisfied if the production set exhibits a vertical tangent, that is when the marginal productivity of the input is infinite. In Vohra (1992), this idea is formalized in a general setting but whith the same a priori given sets of inputs and outputs for all firms.

The aim of this article is to provide a condition on the firms individual pricing behaviour under which the aggregate survival is ensured for the marginal pricing rule as well as for general pricing rules. We first states this condition in an abstract framework with a partition of the set of commodities. We provide a first interpretation in terms of degree of sophistication of the commodities.

The most interesting application of our result concerns intertemporal economies. Indeed, we then consider the the economically meaningful partition given by the time structure of the economy. Our result holds true with an arbitrary number of periods and it opens the way to infinite horizon models through a limit argument. Furthermore, we now have a tractable existence result, which opens the possibility to apply it in dynamical macroeconomic models.

The amazing ascertainment is how mild is our condition in this context. Indeed, for a general pricing rule, it merely requires that if a costly investment is done at one period, there exists a positive return at a future period, that is a least an output with a positive price. For the marginal pricing rule, we consider the case where the production possibilities are defined recursively by a sequence of one-period production mappings at it is done in most of the dynamical models. This just means that production is time consuming. Then, the global production sector satisfies the Survival Assumption if, for each period, the marginal productivity of inputs is bounded above. So, it appears that it is economically not restrictive.

The paper is organized as follows: in Section 2, we briefly present the model of an Arrow-Debreu economy and the representation of the behavior of non-convex producers by pricing rules; Section 3 is devoted to the statement of Assumption $(\mathrm{CH})$ on each producer and we show that it is sufficient to obtain the Survival Assumption at the aggregate level; Section 4 deals with the marginal pricing rule and we state the generalization of the Bounded Marginal Return Assumption associated to a partition of the commodity set; finally, Section 5 studies the case of intertemporal economies and provides the adaptation of Assumptions $(\mathrm{CH})$ and $(\mathrm{BMR})$ to the natural partition 
induced by the sequence of periods.

\section{$2 \quad$ The model}

We consider an economy with positive finite numbers $\ell$ of commodities and $n$ of firms. We take $\mathbb{R}^{\ell}$ for commodity space, and we consider normalized price vectors in $S$, the unit simplex of $\mathbb{R}^{\ell}$.

The technological possibilities of firm $j,(j=1, \ldots, n)$ are represented by a subset $Y_{j} \subset \mathbb{R}^{\ell}$ satisfying the following assumption:

Assumption (P) For every $j, Y_{j}$ is a closed subset of $\mathbb{R}^{\ell}$ satisfying the free-disposal property: $Y_{j}-\mathbb{R}_{+}^{\ell} \subset Y_{j}$ and the possibility of inaction: $0 \in Y_{j}$.

Note that, under Assumption (P), for every $j$, the boundary $\partial Y_{j}$ of the production set $Y_{j}$ exactly coincides with the set of (weakly) efficient productions of firm $j$. For every $j$, the behavior of firm $j$ is described by its pricing rule $\varphi_{j}$, a correspondence which associates with each (weakly) efficient production $y_{j}$ a subset $\varphi_{j}\left(y_{j}\right)$ of $S$ of admissible price vectors. This formalization is compatible with various behaviors considered in the economic literature, notably with the profit maximization, the average pricing or the marginal pricing, defined for producer $j$, respectively, by:

$$
\begin{gathered}
P M_{j}\left(y_{j}\right)=\left\{p \in S \mid p \cdot y_{j} \geq p \cdot y_{j}^{\prime} \text { for every } y_{j} \in Y_{j}\right\}, \\
A P_{j}\left(y_{j}\right)=\left\{p \in S \mid p \cdot y_{j}=0\right\}, \\
M P_{j}\left(y_{j}\right)=N_{Y_{j}}\left(y_{j}\right) \cap S,
\end{gathered}
$$

where $N_{Y_{j}}\left(y_{j}\right)$ denotes the Clarke's normal cone to $Y_{j}$ at $y_{j}$ (See Clarke 1983, Cornet 1988). For every $j$, firm $j$ is said to be in equilibrium at the pair $\left(p, y_{j}\right) \in S \times \partial Y_{j}$ if $p$ is an admissible price vector for firm $j$ given the (weakly) efficient production $y_{j}$, i.e. if $p \in \varphi_{j}\left(y_{j}\right)$. Given the productions $\left(y_{j}\right) \in \prod_{j=1}^{n} \partial Y_{j}$, if there exists a price vector $p \in S$ admissible for every firm $j$, then we say that the collection $\left(p,\left(y_{j}\right)\right)$ is a production equilibrium. We shall denote by $P E$ the set of production equilibria of the economy, that is:

$$
P E=\left\{\left(p,\left(y_{j}\right)\right) \in S \times \prod_{j=1}^{n} \partial Y_{j} \mid p \in \varphi_{j}\left(y_{j}\right) \text { for every } j\right\} .
$$


Since our main focus is on the production sector of the economy, we just consider for the consumption sector the agregate consumption set $X \subset \mathbb{R}^{\ell}$ and the agregate initial endowments $\omega \in \mathbb{R}^{\ell}$. We make the following assumption on the consumption side:

Assumption (C) $X=\mathbb{R}_{+}^{\ell}$ and $\omega_{h}>0$ for all $h=1, \ldots, \ell$.

\section{An alternative to the survival assumption}

It is quite justified to wonder about the appropriateness of the survival assumption since it poses a condition on endogenous variables, the production equilibria. In Bonnisseau and Cornet (1991), existence of equilibria is proven under the following version of the survival assumption :

Assumption (SA) For every $\left(p,\left(y_{j}\right)\right) \in P E$,

$$
p \cdot\left(\sum_{j=1}^{n} y_{j}+\omega\right)>\min \left\{0, \min \left\{\sum_{j=1}^{n} y_{j h}+\omega_{h} \mid h=1, \ldots, \ell\right\}\right\} .
$$

Since $p \in S$, one always has

$$
p \cdot\left(\sum_{j=1}^{n} y_{j}+\omega\right) \geq \min \left\{\sum_{j=1}^{n} y_{j h}+\omega_{h} \mid h=1, \ldots, \ell\right\} .
$$

Hence Assumption (SA) is satisfied when the productions $\left(y_{j}\right)$ is strictly feasible in the sense that $\sum_{j=1}^{n} y_{j h}+\omega_{h}>0$ for all $h$. Otherwise, Assumption $(\mathrm{SA})$ only requires to have a strict inequality $p \cdot\left(\sum_{j=1}^{n} y_{j}+\omega\right)>$ $\min \left\{\sum_{j=1}^{n} y_{j h}+\omega_{h} \mid h=1, \ldots, \ell\right\}$ instead of a weak inequality. So, Assumption (SA) means that the firms following independently their pricing rules do not select the worse possible joint price $p$ in terms of global wealth. Note that this assumption is satisfied if the firms follow loss-free pricing rules, that is if $q \cdot y_{j} \geq 0$ for all $q \in \varphi_{j}\left(y_{j}\right)$.

Before stating the sufficient conditions on the general pricing rules, we shall introduce several notations. For every $j$ and every (weakly) efficient 
production $y_{j} \in \partial Y_{j}$, we let $O\left(y_{j}\right)$ be the set of $y_{j}$-outputs, that is the subset of commodities $h,(h=1, \ldots, \ell)$ satisfying $y_{j h} \geq 0$, and $I\left(y_{j}\right)=\{1, \ldots, \ell\} \backslash$ $O\left(y_{j}\right)$ the set of $y_{j}$-inputs. Note that the possibility of inaction and the freedisposal assumption imply that $0\left(y_{j}\right)$ is nonempty for all weakly-efficient production $y_{j}$.

Our hierarchical condition involves a partition of the set of commodities. So, for any $H \subset\{1, \ldots, \ell\}, z^{H}$ is the vector of $\mathbb{R}^{H}$ with the coordinates of the vector $z$ for the commodities $h \in H$. For any partition $\mathcal{H}=\left(H_{1}, \ldots, H_{k}\right)$ of the commodity set $\{1, \ldots, \ell\}$, for every commodity $h$, we let $\nu(h)$ be the unique integer in $\{1, \ldots, k\}$ satisfying $h \in H_{\nu(h)}$.

Assumption (CH) There exists a partition $\mathcal{H}=\left(H_{1}, \ldots, H_{k}\right)$ of the commodity set $\{1, \ldots, \ell\}$, such that, for every $j$, every $y_{j} \in \partial Y_{j}$, every $q_{j} \in \varphi_{j}\left(y_{j}\right)$ and every $h \in I\left(y_{j}\right)$, if $q_{j h}>0$, then there exists a commodity $k$ such that $\nu(k)>\nu(h)$ and $q_{j k}>0$.

For a pair $\left(q_{j}, y_{j}\right)$, if it does not exist a commodity $h \in I\left(y_{j}\right)$ with $q_{j h}>0$, then Assumption (CH) is obviously satisfied.

We remark that Assumption ( $\mathrm{CH}$ ) involves only fundamentals of the economy, namely production sets and pricing rules and it does not involve the set of production equilibria, which is endogenous. Assumption $(\mathrm{CH})$ can be checked independently for each producer. This is the major improvement with respect to the standard survival assumption.

To interpret this assumption, we remark that the partition $\mathcal{H}$ induces a hierarchy among the commodities. The higher is $\nu(h)$, the higher is the commodity $h$ in the hierarchy. Then, Assumption $(\mathrm{CH})$ means that if an input has a positive price, then there exists another commodity with a positive price, which is higher in the hierarchy. By a recursive argument, this implies that there exists an output with a positive price, which is higher in the hierarchy than an input with a positive price. In particular, this implies that the commodities in the highest class $H_{k}$ are either outputs or free inputs in the sense that the price is equal to 0.

A first example of a hierarchy given by a partition $\mathcal{H}$ of the commodity set is associated to a degree of sophistication. We can rank the commodities from the raw materials to the consumption goods, which are not used in the production of other commodities. In that case, Assumption $(\mathrm{CH})$ means that if the firm chooses a price $q_{j}$ associated to the production $y_{j}$ according to 
the pricing rule $\varphi_{j}$, then if the price of an input is positive, there exists an output with a positive price and a degree of sophistication higher than the one of the input.

Globally, the production is oriented towards more sophisticated commodities. This leads to a weak coordination of the production sector which avoids the worse possible choice of the price when each firm follows its pricing rule.

Proposition 1 Assumption (SA) is satisfied if Assumptions (C), (P) and (CH) hold true.

A fundamental consequence of this proposition is the fact that we can get the existence of an equilibrium with general pricing rules under standard assumptions on the consumer side of the economy, Assumption $(\mathrm{P})$ on the production side, the bounded losses Assumption and Assumption (CH) replacing Assumption (SA). So, we have an existence result under assumptions only on the fundamentals of the economy like for a competitive equilibrium in the Arrow-Debreu theory.

Proof: Let us assume by contraposition that there exists $\left(p,\left(y_{j}\right)\right) \in P E$ such that $p \cdot\left(\sum_{j=1}^{n} y_{j}+\omega\right) \leq \min \left\{0, \min _{h}\left\{\sum_{j=1}^{n} y_{j h}+\omega_{h}\right\}\right\}$. Since $p \in S$, $p \cdot\left(\sum_{j=1}^{n} y_{j}+\omega\right) \geq \min _{h}\left\{\sum_{j=1}^{n} y_{j h}+\omega_{h}\right\}$. Hence, one gets

$$
p \cdot\left(\sum_{j=1}^{n} y_{j}+\omega\right)=\min _{h}\left\{\sum_{j=1}^{n} y_{j h}+\omega_{h}\right\} \leq 0
$$

This implies that $\sum_{j=1}^{n} y_{j h^{\prime}}+\omega_{h^{\prime}}=\min _{h}\left\{\sum_{j=1}^{n} y_{j h}+\omega_{h}\right\}$ for every commodity $h^{\prime}$ such that $p_{h^{\prime}}>0$. From Assumption (C), $\omega_{h}>0$ for all $h$, hence if $p_{h}>0$ then $\sum_{j=1}^{n} y_{j h}<0$.

Since $p \in S$, there exists $h_{0}$ such that $p_{h_{0}}>0$. Since $\sum_{j=1}^{n} y_{j h_{0}}<0$, there exists $j_{0}$ such that $y_{j_{0} h_{0}}<0$. Let $\bar{h}_{0} \in\{1, \ldots, \ell\}$ such that $p_{\bar{h}_{0}}>0, y_{j_{0} \bar{h}_{0}}<0$ and $\nu\left(\bar{h}_{0}\right) \geq \nu(h)$ for all $h$ satisfying $p_{h}>0$ and $y_{j_{0} h}<0$. Such $\bar{h}_{0}$ exists since the set of commodities is finite.

Assumption $(\mathrm{CH})$ implies that there exists $h_{1}$ such that $p_{h_{1}}>0$ and $\nu\left(h_{1}\right)>\nu\left(\bar{h}_{0}\right)$. The choice of $\bar{h}_{0}$ implies that $y_{j_{0} h_{1}} \geq 0$. Consequently, since $\sum_{j=1}^{n} y_{j h_{1}}<0$, there exists $j_{1}$ such that $y_{j_{1} h_{1}}<0$. With the same argument, we find a commodity $\bar{h}_{1}$ such that $\nu\left(\bar{h}_{1}\right) \geq \nu\left(h_{1}\right)>\nu\left(\bar{h}_{0}\right)$. By iterating this procedure, one gets a contradiction since the number of commodities is finite. 


\section{The case of the marginal pricing rule}

With the marginal pricing rule, we can be more precise and give a condition on the production sets under which Assumption $(\mathrm{CH})$ holds true. This more primitive condition is a condition of bounded marginal returns. Indeed, in a single firm and two commodity economy, the Survival Assumption may not be satisfied if the boundary of the production set has a vertical tangent. Such a tangency implies that the marginal productivity is infinite. We show that, even if they are increasing, marginal returns must be bounded above for the Survival Assumption to be satisfied.

If the production set $Y_{j}$ is defined by a smooth transformation function $g_{j}$ on a neighborhood of a weakly efficient production $y_{j}$ that is, for a neighborhood $U$ of $y_{j}, Y_{j} \cap U=\left\{z_{j} \in U \mid g_{j}\left(z_{j}\right) \leq 0\right\}$, then the marginal return of an input $h_{1}$ for an output $h_{2}$ is

$$
\frac{\frac{\partial g_{j}}{\partial y_{h_{1}}}\left(y_{j}\right)}{\frac{\partial g_{j}}{\partial y_{h_{2}}}\left(y_{j}\right)}
$$

So, the marginal return is bounded if $\frac{\partial g_{j}}{\partial y_{h_{2}}}\left(y_{j}\right)$ is positive. This means that, locally, the level of production in commodity $h_{2}$ is a smooth function of quantities of inputs.

To capture this idea without smoothness assumption on the production set, we can remark that the above condition implies that there exists an inward direction ${ }^{2} z$ at $y_{j}$, satisfying $z_{h}>0$ for all $h \neq h_{2}$. So, we will put our assumption on the set of inward directions, that is on the tangent cone.

We recall that the Clarke's tangent cone to $Y_{j}$ at $y_{j}$ is defined by:

$$
T_{Y_{j}}\left(y_{j}\right)=\left\{\begin{array}{l|l}
v \in \mathbb{R}^{\ell} & \begin{array}{l}
\forall\left(y_{j}^{\nu}\right) \subset Y_{j},\left(y_{j}^{\nu}\right) \rightarrow y_{j}, \\
\forall\left(t^{\nu}\right) \subset \mathbb{R}_{+},\left(t^{\nu}\right) \rightarrow 0, \exists\left(v^{\nu}\right) \subset \mathbb{R}^{\ell} \\
\text { such that }\left(v^{\nu}\right) \rightarrow v \text { and } y_{j}^{\nu}+t^{\nu} v^{\nu} \in Y_{j} \forall \nu
\end{array}
\end{array}\right\} .
$$

From the free-disposal assumption, we may have some useless inputs for the production set. These inputs are not relevant in the production process and they have a zero price when the firm follows the marginal pricing rule.

\footnotetext{
${ }^{2}$ That is, $y_{j}+t z \in Y_{j}$ for all $t>0$ small enough. In the smooth case, if $\nabla g_{j}\left(y_{j}\right) \cdot z<0$, then $z$ is inward.
} 
Definition 1 Let us consider a production set $Y_{j}$ satisfying Assumption $(P)$. A commodity $h$ is a useless input if for all $y_{j} \in \partial Y_{j}, y_{j}^{\prime}=\left(\left(y_{j k}\right)_{k \neq h}, 0\right)$ belongs to $Y_{j}$ whenever $y_{j h}<0$.

The following lemma recall that a useless input has a zero price when the firm follows the marginal pricing rule. The proof is given in Appendix.

Lemma 1 Let us consider a production set $Y_{j}$ satisfying Assumption $(P)$. Let $h$ be a useless input. Then, for all $y_{j} \in \partial Y_{j}$ and $q \in M P_{j}\left(y_{j}\right)$, if $y_{j h}<0$, then $q_{h}=0$.

In the following, $\tilde{I}\left(y_{j}\right)$ is the set of non-useless input and, for any partition $\mathcal{H}=\left(H_{1}, \ldots, H_{k}\right)$ of the commodity set, $\bar{\nu}\left(y_{j}\right)=\max \left\{\nu(h) \mid h \in \tilde{I}\left(y_{j}\right)\right\}$.

We can now state our assumption on the bounded marginal return of $Y_{j}$, which implies that Assumption $(\mathrm{CH})$ holds true for the marginal pricing rule.

Assumption (BMR) There exists a partition $\mathcal{H}=\left(H_{1}, \ldots, H_{k}\right)$ of the commodity set $\{1, \ldots, \ell\}$, such that, for every $j$, every $y_{j} \in \partial Y_{j}$, there exists $z \in T_{Y_{j}}\left(y_{j}\right)$ such that $z_{h}>0$ for all $h \in \tilde{I}\left(y_{j}\right)$ and $z_{h^{\prime}} \geq 0$ for all $h^{\prime} \in$ $\cup_{\nu \leq \bar{\nu}\left(y_{j}\right)} H_{\nu}$.

The interpretation of Assumption (BMR) is the following. From the freedisposal assumption, a vector in the tangent cone cannot be positive. So, if we consider the vector $z$ given by Assumption (BMR), the exists an output $k$, satisfying $\nu(k)>\bar{\nu}\left(y_{j}\right)$ and $z_{k}<0$. If $z$ is an inward direction, then $-z$ is an outward direction. So, for $t$ small enough, $y_{j}-t z$ does not belongs to $Y_{j}$. This means that the increase of the quantities of inputs $\left(t z_{h}\right)_{h \in \tilde{I}\left(y_{j}\right)}$ is not enough to produce the additional quantity of output $-t z_{k}>0$. Hence, the productivity of the inputs is bounded above.

To compare with Vohra (1992), we just have to note that the assumption in this paper involves only a two set partition with the upper class being the one of "final" outputs, i.e., commodities that are never used as an input by any firm. Vohra's assumption rules out the possibility of an intermediate producer, in the sense that it only produces commodities that will be inputs for others firms since his Bounded Marginal Return Assumption implies that each firm is able to produce at least one final output. 
Proposition 2 Under Assumptions (C) and (P), Assumption (BMR) implies that Assumption ( $\mathrm{CH}$ ) holds for the marginal pricing rule.

An immediate corollary of this proposition and Proposition 1 is that the survival assumption is a consequence of Assumptions (C), (P) and (BMR) for the marginal pricing rule. So, as for general pricing rules, we obtain the existence of a marginal pricing equilibrium under assumptions only on the fundamentals of the economy.

Proof: Let $y_{j} \in \partial Y_{j}$ and $q_{j} \in M P_{j}\left(y_{j}\right)$. Since the normal cone is the negative polar cone of the tangent cone, one has $q_{j} \cdot z \leq 0$ for the vector $z$ given by Assumption (BMR). Furthermore, for all useless inputs $k$, if $y_{j k}<0$, then the vector with zero components but the $k$ th one equal to 1 belongs to the tangent cone $T_{Y_{j}}\left(y_{j}\right)$. This implies that $q_{j k}=0$.

Let a commodity $h$ such that $y_{j h}<0$ and $q_{j h}>0$. Consequently, $h$ is not a useless input and $\nu(h) \leq \bar{\nu}\left(y_{j}\right)$. Let $z$ as given by Assumption (BMR). Then,

$$
\sum_{h^{\prime} \mid \nu\left(h^{\prime}\right) \leq \bar{\nu}\left(y_{j}\right)} q_{j h^{\prime}} z_{h^{\prime}}>0 .
$$

Since $q_{j} \cdot z \leq 0$, there exists $\bar{h}$ such that $\nu(\bar{h})>\bar{\nu}\left(y_{j}\right) \geq \nu(h)$ such that $z_{\bar{h}}<0$ and $q_{j \bar{h}}>0$. Hence Assumption $(\mathrm{CH})$ holds true.

\section{The survival assumption in an intertempo- ral economy}

We now consider the most important application of the general result given above, namely, an intertemporal economy. Indeed, in this case, the partition of the set of commodities is natural: it is defined by the date at which the commodity is available. This case is fundamental since it allows us to consider the existence of equilibrium in a dynamical model with a finite horizon and in an overlapping generation model. We can then expect to consider an infinite horizon model through a limit argument.

We are considering an economy with $T$ periods and $\ell$ commodities at each period. Then the commodity space is $\mathbb{R}^{\ell T}$ and each commodity is represented by a pair $(h, t)$ in $\{1, \ldots, \ell\} \times\{1, \ldots, T\}$. In this setting, the set 
of commodities is naturally partitioned by the date at which the commodity is available. So we have a natural partition $\mathcal{H}=\left(H_{1}, \ldots, H_{T}\right)$. For each commodity $(h, t)$, the index $\nu(h, t)$ defined in the previous section is merely equal to $t$, that is the date at which the commodity $h$ is available.

In this framework, we can rewrite Assumption $(\mathrm{CH})$ as follows:

Assumption (CT)For every $j$, every $y_{j} \in \partial Y_{j}$, every $q_{j} \in \varphi_{j}\left(y_{j}\right)$ and every commodity $(h, t) \in I\left(y_{j}\right)$, if $q_{j(h, t)}>0$, then there exists a commodity $(k, \tau)$ such that $\tau>t$ and $q_{j(k, \tau)}>0$.

This assumption is really mild since it simply claims that a costly investment $q_{j(h, t)} y_{j(h, t)}<0$ at a period $t$ is done only if there is a possibility of a non negative return in a subsequent period $q_{j(k, \tau)} y_{j(k, \tau)} \geq 0$. If the production is time consuming in the sense that an investment at one period produces some outputs only in the next periods, the statement is totally intuitive since no return can be expected at the period of investment. An economically reasonable pricing rule should satisfy this requirement, which is much more lighter than a no-loss condition, which requires that the return is larger than the investment.

So, in a multi-period economy with increasing returns, the existence of a general economic equilibrium with pricing rules can be obtained under the standard assumptions as the ones presented in Bonnisseau and Cornet (1991) or Villar (1999) but Assumption (CT) replacing the Survival Assumption. Hence, the class of pricing rules compatible with an equilibrium is very large and far beyond the no-loss pricing rules.

We now turn to the case of the marginal pricing rule with the production possibilities represented by production mappings as it is done in most of the dynamical economic models.

A production mapping is a set-valued mapping $F$ from $-\mathbb{R}_{+}^{\ell}$ to $\mathbb{R}^{\ell}$. For a given vector of inputs $z \in-\mathbb{R}_{+}^{\ell}, F(z)$ is the set of possible outputs. We posit the following assumption on $F$.

Assumption (F) $F$ has a closed graph, nonempty values, $F(0)=-\mathbb{R}_{+}^{\ell}$, for all $z \in-\mathbb{R}_{+}^{\ell}, F(z) \cap \mathbb{R}_{+}^{\ell}$ is bounded, $F(z)=\left(F(z) \cap \mathbb{R}_{+}^{\ell}\right)-\mathbb{R}_{+}^{\ell}$ and for all $\left(z, z^{\prime}\right) \in\left(-\mathbb{R}_{+}^{\ell}\right)^{2}$, if $z \leq z^{\prime}$, then $F\left(z^{\prime}\right) \subset F(z)$.

Assumption $(\mathrm{F})$ is standard. It incorporates the free-disposability in the production process and the fact that the production process takes time in 
the sense that the consumption of an input at a period $t$ has no influence on the output at this period. To a production mapping $F$, we associate an elementary production set $Z$ in $\left(\mathbb{R}^{\ell}\right)^{2}$ defined by:

$$
Z=\left\{(z, \zeta) \in-\mathbb{R}_{+}^{\ell} \times \mathbb{R}^{\ell} \mid \zeta \in F(z)\right\}
$$

For the intertemporal production, we assume that the production possibilities of a producer $j$ at a period $t$ is represented by a production mapping $F_{j t}$ or by the associated production set $Z_{j t}$. To describe the global production possibilities on the $T$ periods, we use the following linear mapping $\Gamma$ from $\left(\mathbb{R}^{\ell} \times \mathbb{R}^{\ell}\right)^{T-1}$ to $\left(\mathbb{R}^{\ell}\right)^{T}$ defined by:

$$
\Gamma\left(\left(z_{t}, \zeta_{t+1}\right)_{t=1}^{T-1}\right)=\left(z_{1},\left(z_{t}+\zeta_{t}\right)_{t=2}^{T-1}, \zeta_{T}\right)
$$

The production set is then :

$$
Y_{j}=\Gamma\left(\prod_{t=1}^{T-1} Z_{j t}\right)
$$

One checks that Assumption $(\mathrm{P})$ is satisfied by $Y_{j}$ when the production mappings $F_{j t}$ satisfy Assumption $(\mathrm{F})$.

Now, we proved that if the production mappings $F_{j t}$ satisfy the bounded marginal return condition then the global production set $Y_{j}$ satisfies Assumption (CT) for the marginal pricing rule. We can now state our result.

Proposition 3 Under Assumptions $(C)$ and $(F)$, if the elementary production sets $Z_{j t}$ satisfy Assumption (BMR) for the natural partition associated to the two periods, then the production set $Y_{j}$ satisfies Assumption (CT) for the marginal pricing rule.

Using this result, one remarks that we can check directly on the elementary production sets $Z_{j t}$ if the whole production set $Y_{j}$ satisfies Assumption (CT) and the result is independent of the number of periods. On $Z_{j t}$, since the partition has only two elements, we are in the same setting as Vohra (1992). Our contribution is to show that we can extend the result from a two-period economy to a $T$-period economy.

Furthermore, Assumption (BMR) has a simpler formulation for an elementary production set $Z_{j t}$. Indeed, the commodities in the second period 
are either outputs or useless inputs. Hence Assumption (BMR) holds true if for every $\left(z_{j}, \zeta_{j}\right) \in \partial Z_{j}$, there exists $(z, \zeta) \in T_{Z_{j}}\left(z_{j}, \zeta_{j}\right)$ such that $z \geq 0$ and $z_{h}>0$ if $z_{j h}<0$.

Proof of Proposition 3. We first recall some definitions of normal cones that we will use in the following.

Definition 2 Let $X$ be a subset of $\mathbb{R}^{n}$ and $x$ an element of $X$. Let $\|x\|$ be the canonic Euclidean norm of $x$.

(a) The proximal normal cone to $X$ at $x$, denoted $N_{X}^{P}(x)$, is defined by:

$$
N_{X}^{P}(x)=\left\{v \in \mathbb{R}^{n} \mid \exists \alpha>0, B(x+\alpha v, \alpha\|v\|) \cap X=\emptyset\right\}
$$

(b) The limiting normal cone to $X$ at $x$, denoted $N_{X}^{L}(x)$, is defined by:

$$
N_{X}(x)=\left\{\begin{array}{l|l}
y \in \mathbb{R}^{n} & \begin{array}{l}
\exists\left(x^{\nu}, y^{\nu}\right) \subset X \times \mathbb{R}^{n},\left(x^{\nu}, y^{\nu}\right) \rightarrow(x, y), \\
y^{\nu} \in N_{X}^{P}\left(x^{\nu}\right), \forall \nu \in \mathbb{N}
\end{array}
\end{array}\right\}
$$

(c) The Clarke's normal to $X$ at $x$, denoted $N_{X}(x)$ is closed convex hull of $N_{X}^{L}(x)$.

We now state a lemma, the proof of which is given in Appendix, on the relation between the limiting normal cone of $Y_{j}$ and the limiting normal cones of the elementary production set $Z_{j}$.

Lemma 2 Let $q$ an element of $N_{Y_{j}}^{L}\left(y_{j}\right)$. Then, under Assumption $(F)$, there exists $\left(z_{j, t}, \zeta_{j, t+1}\right)_{t=1}^{T-1} \in \prod_{t=1}^{T-1} Z_{j t}$ such that

(i) $\left.y_{j}=\Gamma\left(\left(z_{j, t}, \zeta_{j, t+1}\right)_{t=1}^{T-1}\right)\right)$;

(ii) for all $t=1, \ldots, T-2, \zeta_{j, t+1} \geq 0$;

(iii) for all $t=1, \ldots, T-1,\left(q_{j, t}, q_{j, t+1}\right) \in N_{Z_{j t}}^{L}\left(z_{j, t}, \zeta_{j, t+1}\right)$.

We now come to the proof of Proposition 3. Let $y_{j} \in \partial Y_{j}, q_{j} \in M P_{j}\left(y_{j}\right)$ and $(h, \tau)$ such that $y_{j(h, \tau)}<0$ and $q_{j(h, \tau)}>0$. 
Under Assumption (P), the limiting normal cone is a closed cone included in $\mathbb{R}_{+}^{\ell T}$. Hence its closed convex hull is merely the convex hull. So, there exists $\left(q_{j}^{1}, \ldots, q_{j}^{k}\right) \in\left(N_{Y_{j}}^{L}\left(y_{j}\right)\right)^{k}$ and $\beta \in \mathbb{R}_{++}^{k}$ such that $q_{j}=\sum_{\kappa=1}^{k} \beta^{\kappa} q_{j}^{\kappa}$. Consequently, there exists at least an index $\kappa$ such that $q_{j(h, \tau)}^{\kappa}>0$. From Lemma 2, there exists $\left(z_{j, t}, \zeta_{j, t+1}\right)_{t=1}^{T-1} \in \prod_{t=1}^{T-1} Z_{j t}$ such that

(i) $\left.y_{j}=\Gamma\left(\left(z_{j, t}, \zeta_{j, t+1}\right)_{t=1}^{T-1}\right)\right)$;

(ii) for all $t=1, \ldots, T-2, \zeta_{j, t+1} \geq 0$;

(iii) for all $t=1, \ldots, T-1,\left(q_{j, t}^{\kappa}, q_{j, t+1}^{\kappa}\right) \in N_{Z_{j t}}^{L}\left(z_{j, t}, \zeta_{j, t+1}\right)$.

Since $q_{j(h, \tau)}^{\kappa}>0$ and $y_{j(h, t)}<0$, one first remarks that $\tau<T$. Indeed, if $\tau=T$, then $\left(q_{j, T-1}^{\kappa}, q_{j, T}^{\kappa}\right) \in N_{Z_{j, T-1}}^{L}\left(z_{j, T-1}, \zeta_{j, T}\right) \subset N_{Z_{j, T-1}}\left(z_{j, T-1}, \zeta_{j, T}\right)$ and $\zeta_{j(h, T)}=y_{j(h, T)}<0$. From the definition of $Z_{j, T-1}$ through the production mapping $F_{j, T-1}$ satisfying Assumption $(\mathrm{F})$, one deduces that the commodity $(T, h)$ is a useless input of $Z_{j, T-1}$, which satisfies Assumption (P). So, from Lemma $1, q_{j(h, T)}^{\kappa}=0$, which contradicts $q_{j(h, T)}^{\kappa}=q_{j(h, \tau)}^{\kappa}>0$.

Since $y_{j(h, \tau)}<0$ and $\zeta_{j, \tau} \geq 0$, one deduces that $z_{j, h, \tau)}<0$. Since, $Z_{j, \tau}$ satisfies Assumption (BMR), Proposition 2 shows that $Z_{j, \tau}$ satisfies Assumption $(\mathrm{CH})$ for the marginal pricing rule. Consequently, since $\left(q_{j, \tau}^{\kappa}, q_{j, \tau+1}^{\kappa}\right) \in$ $N_{Z_{j \tau}}^{L}\left(z_{j, \tau}, \zeta_{j, \tau+1}\right) \subset N_{Z_{j \tau}}\left(z_{j, \tau}, \zeta_{j, \tau+1}\right)$, there exists a commodity $h^{\prime}$ such that $q_{j,\left(h^{\prime}, \tau+1\right)}^{\kappa}>0$. Since for all $\kappa^{\prime}, q_{j,\left(h^{\prime}, \tau+1\right)}^{\kappa^{\prime}} \geq 0$, one concludes that $q_{j,\left(h^{\prime}, \tau+1\right)}>0$. So, $Y_{j}$ satisfies Assumption $(\mathrm{CT})$ for the marginal pricing rule.

\section{Appendix}

Proof of Lemma 1. Let $y_{j} \in \partial Y_{j}$ and $q \in M P_{j}\left(y_{j}\right)$ such that $y_{j h}<0$. From the definitions of a useless input and the tangent normal cone, one easily shows that the vector $\varepsilon^{h}$, the vector with 0 components but the $h$ th one equal to 1 , belongs to the tangent cone $T_{Y_{j}}\left(y_{j}\right)$. Since the Clarke's normal cone is the negative polar cone of the tangent cone, one deduces that $q_{h} \leq 0$. Since $Y_{j}$ satisfies the free-disposal Assumption, then $q_{h} \geq 0$. So, $q_{h}=0$.

Proof of Lemma 2. Let $y_{j} \in \partial Y_{j}$ and $q \in N_{Y_{j}}^{L}\left(y_{j}\right)$. From the definition of the limiting normal cone, there exists a sequence $\left(y_{j}^{\nu}\right)$ in $\partial Y_{j}$, which converges 
to $y_{j}$ and a sequence $\left(q^{\nu}\right)$, which converges to $q$ such that $q^{\nu} \in N_{Y_{j}}^{P}\left(y_{j}^{\nu}\right)$ for all $\nu$.

The definition of $Y_{j}$ implies that there exists $\left(\left(z_{j, t}^{\nu}, \zeta_{j, t+1}^{\nu}\right)_{t=1}^{T-1}\right) \in \prod_{t=1}^{T-1} Z_{j t}$ such that $\left.y_{j}^{\nu}=\Gamma\left(\left(z_{j, t}^{\nu}, \zeta_{j, t+1}^{\nu}\right)_{t=1}^{T-1}\right)\right)$ for all $\nu$. We can assume that $\zeta_{j, t+1}^{\nu} \geq 0$ for all $t=1, \ldots, T-2$. If not, we replace $z_{j, t}^{\nu}$ by $z_{j, t}^{\nu}+\zeta_{j, t+1}^{\nu}-\zeta_{j ; t+1}^{\nu+}$ and $\zeta_{j, t+1}^{\nu}$ by $\zeta_{j ; t+1}^{\nu+}$, where $\zeta_{j ; t+1}^{\nu+}$ is the projection of $\zeta_{j, t+1}^{\nu}$ on $\mathbb{R}_{+}^{\ell}$. From Assumption (F), since $z_{j, t}^{\nu}+\zeta_{j, t+1}^{\nu}-\zeta_{j ; t+1}^{\nu+} \leq z_{j, t}^{\nu}$, one checks that $\zeta_{j ; t+1}^{\nu+} \in F_{j, t}\left(z_{j, t}^{\nu}+\zeta_{j, t+1}^{\nu}-\zeta_{j ; t+1}^{\nu+}\right)$ for all $t$.

We now prove that the sequence $\left.\left(\left(z_{j, t}^{\nu}, \zeta_{j, t+1}^{\nu}\right)_{t=1}^{T-1}\right)\right)$ is bounded. We first remark that $z_{j, 1}^{\nu}=y_{j, 1}^{\nu}$ and it converges to $y_{j, 1}$, hence it is bounded. Let $\underline{z}_{1}$ be such that $\underline{z}_{1} \leq z_{j, 1}^{\nu}$ for all $\nu$. If $T+2$, then $\left(\zeta_{j, 2}^{\nu}=y_{j, 2}^{\nu}\right)$ and it is bounded since $\left(y_{j, 2}^{\nu}\right)$ converges to $y_{j, 2}$. If $T>2$, for all $\nu, \zeta_{j, 2}^{\nu} \in F_{j, 1}\left(z_{j, 1}^{\nu}\right) \subset F_{j, 1}\left(\underline{z}_{1}\right)$. So, from Assumption (F), the sequence $\left(\zeta_{j, 2}^{\nu}\right)$ is bounded since $F_{j, 1}\left(\underline{z}_{1}\right) \cap \mathbb{R}_{+}^{\ell}$ is bounded. Since $\left(z_{j, 2}^{\nu}+\zeta_{j, 2}^{\nu}=y_{j, 2}^{\nu}\right)$ converges to $y_{j, 2}$, one deduces that the sequence $\left(z_{j, 2}^{\nu}\right)$ is bounded. Using recursively the same argument, we finally show that the sequence $\left.\left(\left(z_{j, t}^{\nu}, \zeta_{j, t+1}^{\nu}\right)_{t=1}^{T-1}\right)\right)$ is bounded.

Since the sequence $\left.\left(\left(z_{j, t}^{\nu}, \zeta_{j, t+1}^{\nu}\right)_{t=1}^{T-1}\right)\right)$ is bounded, it has a converging subsequence that we denote again by $\left.\left(\left(z_{j, t}^{\nu}, \zeta_{j, t+1}^{\nu}\right)_{t=1}^{T-1}\right)\right)$ for the sake of simpler notations. The limit is denoted $\left.\left(\left(z_{j, t}, \zeta_{j, t+1}\right)_{t=1}^{T-1}\right)\right)$. Since the elementary production sets $Z_{j, t}$ are closed and the linear mapping $\Gamma$ is continuous, we immediately show that $\left.\left(z_{j, t}, \zeta_{j, t+1}\right)_{t=1}^{T-1} \in \prod_{t=1}^{T-1} Z_{j t}, y_{j}=\Gamma\left(\left(z_{j, t}, \zeta_{j, t+1}\right)_{t=1}^{T-1}\right)\right)$ and for all $t=1, \ldots, T-2, \zeta_{j, t+1} \geq 0$.

We now show that for all $\nu$, for all $t=1, \ldots, T-1,\left(q_{j, t}^{\nu}, q_{j, t+1}^{\nu}\right) \in$ $N_{Z_{j t}}^{P}\left(z_{j, t}^{\nu}, \zeta_{j, t+1}^{\nu}\right)$. Since $q^{\nu} \in N_{Y_{j}}^{P}\left(y_{j}^{\nu}\right)$, there exists $\alpha^{\nu}>0$ such that $B\left(y_{j}^{\nu}+\right.$ $\left.\alpha^{\nu} q^{\nu}, \alpha^{\nu}\left\|q^{\nu}\right\|\right) \cap Y_{j}=\emptyset$. Let us assume by contraposition, that there exists $\left(z_{j, t}^{\prime}, \zeta_{j, t+1}^{\prime}\right) \in B\left(\left(z_{j, t}^{\nu}, \zeta_{j, t+1}^{\nu}\right)+\alpha^{\nu}\left(q_{t}^{\nu}, q_{t+1}^{\nu}\right), \alpha^{\nu}\left\|\left(q_{t}^{\nu}, q_{t+1}^{\nu}\right)\right\|\right) \cap Z_{j, t}$. Then, $y_{j}^{\prime}=\Gamma\left(\left(z_{j, \tau}^{\nu}, \zeta_{j, \tau+1}^{\nu}\right)_{\tau \neq t},\left(z_{j, t}^{\prime}, \zeta_{j, t+1}^{\prime}\right)\right)$ belongs to $Y_{j}$. Furthermore,

$$
y_{j}^{\prime}-y_{j}^{\nu}-\alpha^{\nu} q^{\nu}=\left(\left(-\alpha^{\nu} q_{\tau}^{\nu}\right)_{\tau \neq t, t+1},\left(z_{j, t}^{\nu}, \zeta_{j, t+1}^{\nu}\right)+\alpha^{\nu}\left(q_{t}^{\nu}, q_{t+1}^{\nu}\right)\right)
$$

So,

$$
\begin{aligned}
\left\|y_{j}^{\prime}-y_{j}^{\nu}-\alpha^{\nu} q^{\nu}\right\|^{2}= & \left(\alpha^{\nu}\right)^{2} \sum_{\tau \neq t, t+1}\left\|q_{\tau}^{\nu}\right\|^{2}+\left\|\left(z_{j, t}^{\nu}, \zeta_{j, t+1}^{\nu}\right)+\alpha^{\nu}\left(q_{t}^{\nu}, q_{t+1}^{\nu}\right)\right\|^{2} \\
& <\left(\alpha^{\nu}\right)^{2} \sum_{\tau=1}^{T}\left\|q_{\tau}^{\nu}\right\|^{2}
\end{aligned}
$$

Consequently, $y_{j}^{\prime}$ belongs to $B\left(y_{j}^{\nu}+\alpha^{\nu} q^{\nu}, \alpha^{\nu}\left\|q^{\nu}\right\|\right) \cap Y_{j}$, which is a contradiction with $B\left(y_{j}^{\nu}+\alpha^{\nu} q^{\nu}, \alpha^{\nu}\left\|q^{\nu}\right\|\right) \cap Y_{j}=\emptyset$. 


\section{References}

[1] Allais, M., Traité d'Economie Pure, (Paris: Imprimerie Nationale, 1953).

[2] Bonnisseau, J.-M. and B. Cornet, "Existence of marginal cost pricing equilibria: the nonsmooth case", International Economic Review 31, 1990, 685-708.

[3] Bonnisseau, J.-M., and B. Cornet, "General equilibrium theory with increasing returns: the existence problem", in Equilibrium Theory and Applications. Proceedings of the Sixth International Symposium in Economic Theory and Econometrics, (Cambridge: Cambridge University Press, 1991), 65-82.

[4] Brown, D. J., "Equilibrium analysis with nonconvex technologies," in W. Hildenbrand and H. Sonnenschein, eds., Handbook of Mathematical Economics, vol. IV, (Amsterdam: North-Holland, 1991), ch. 36.

[5] Clarke, F., Optimization and Nonsmooth Analysis, (New-York : John Wiley, 1983).

[6] Cornet, B., "General equilibrium theory and increasing returns: Presentation", Journal of Mathematical Economics 17, 1988, 103-118.

[7] Guesnerie, R., "Pareto optimality in non-convex economies", Econometrica 43, 1975, 1-29.

[8] Hotelling, H., "The general welfare in relation to problems of taxation and of railway and utility rates", Econometrica 4, 1938, 242-269.

[9] Lange, O., 1936-1937 "On the economic theory of socialism (parts one and two)", Review of Economic Studies 4, 1936 and 1937, 53-71 and 123-140.

[10] Lerner, A. P., "A note on socialist economics", Review of Economic Studies 4, 1936, 72-76.

[11] Pigou, A. C., The Economics of Welfare, (London: Macmillan, 1932).

[12] Rockafellar, R.T., and R.J-B. Wets, Variational Analysis, (Berlin: Springer-Verlag, 1998). 
[13] Villar, A., 1999. Equilibrium and Efficiency in Production Economies, Lecture Notes in Economics and Mathematical Systems (Berlin: Springer-Verlag, 1999).

[14] Vohra, R., "Marginal cost pricing under bounded marginal returns", Econometrica 60, 1992, 859-876.

[15] Walras, L., Elements d'Economie Politique Pure, (Lausanne: Corbaz, 1874). 